\title{
The use of gold nanoparticle aggregation for DNA computing and logic-based biomolecular detection
}

\author{
In-Hee Lee ${ }^{1} \ddagger$, Kyung-Ae Yang ${ }^{1,2} \ddagger$, Ji-Hoon Lee ${ }^{2}$, Ji-Yoon Park ${ }^{3}$, \\ Young Gyu Chai ${ }^{3}$, Jae-Hoon Lee ${ }^{4} \oint$ and Byoung-Tak Zhang ${ }^{1,2} \|$ \\ ${ }^{1}$ School of Computer Science and Engineering, Seoul National University, 599 \\ Gwanak-ro, Gwanak-gu, Seoul 151-742, Korea \\ ${ }^{2}$ Center for Bioinformation Technology, Seoul National University, 599 Gwanak-ro, \\ Gwanak-gu, Seoul 151-742, Korea \\ ${ }^{3}$ Division of Molecular and Life Sciences, Hanyang University, 1271 Sa-dong, \\ Sangnok-gu, Ansan, Gyeonggi-do 426-791, Korea \\ ${ }^{4}$ Fachgebiet Mikrobiologie und Genetik, Institut für Biotechnologie, Technische \\ Universitaet Berlin, Gustav-Meyer Allee 25, 13355 Berlin, Germany \\ E-mail: btzhang@bi.snu.ac.kr
}

\begin{abstract}
The use of DNA molecules as a physical computational material has attracted much interest, especially in the area of DNA computing. DNAs are also useful for logical control and analysis of biological systems if efficient visualization methods are available. Here we present a quick and simple visualization technique that displays the results of the DNA computing process based on a colorimetric change induced by gold nanoparticle aggregation, and we apply it to the logic-based detection of biomolecules. Our results demonstrate its effectiveness in both DNA-based logical computation and logic-based biomolecular detection.
\end{abstract}

PACS numbers: 87.85.Rs, 64.75.Yz

Submitted to: Nanotechnology

$\ddagger$ These authors contributed equally and want to be addressed as co-first authors.

$\S$ Present address: Digital Genomics, Namsung Plaza, 345-40 Gasan-dong, Geumcheon-gu, Seoul 153782, Korea

|| Corresponding author 


\section{Introduction}

Ever since DNA was used for computing based on molecular recognition affinity [1], it has been applied to various computational problems such as satisfiability problems $[2,3]$, logical game problems $[4,5,6]$, traveling salesman problems [7], maximal clique problems [8], and associative memories [9]. Although the instances tackled by DNA computing are rudimentary when compared with those solved by conventional electronic computers, it has shown potential as a new computing paradigm. DNA computing can also be used as an interface to biological systems for logic-based control of biological events $[10,11,12]$. Biological application requires various advanced technologies including those to monitor processes or to detect outputs produced by DNA computing. In such cases, the conventional detection procedures for DNA computing such as gel electrophoresis and fluorescence spectroscopy may not be appropriate because they are time consuming, laborious and often require expensive instruments.

The aggregation of gold nanoparticles (Au-NPs) shows a visible color change and is controllable by oligonucleotides attached to the Au-NPs [13, 14]. Au-NPs have a wellestablished methodology beyond DNA detection, and the high stability and high activity after conjugating biomolecules make them more attractive than other nanoparticles $[15,16]$. In addition, the detection steps, time, and costs associated with the instruments can be reduced because the color change of Au-NPs is quick and noticeable to the naked eye $[13,14]$. Therefore, Au-NPs can be an alternative tool to visualize DNA computing results. Here, we utilize the color change of Au-NPs in a DNA computing algorithm to solve a logical problem known as resolution theorem proving and to show its potential for logic-based biomolecular detection.

\section{Experimental details}

\subsection{Sequence preparation}

The nucleotide sequences used are shown in table 1. Case A demonstrates the visualization of DNA computing processes using Au-NP aggregation. Case B shows the application to logic-based biomolecular detection. All sequences used in case A were designed using the evolutionary DNA sequence optimization program, Nucleic Acid Computing Simulation Toolkit (NACST) [17] to minimize cross-hybridization and self- or hetero-dimerization. In case B, special care was taken to avoid undesired hybridizations which might be caused by the highly conserved regions in microRNAs (miRNAs) and non-canonical base pairing. The cross-hybridization or non-canonical base pairing, if any, will be highly unstable unless the neighbors form consecutive base pairings, which is very unlikely in our sequence design. To simplify sequence design, we used the same thiol-modified sequences attached to Au-NPs [14] for both cases. All DNA sequences used were purchased from Bioneer (Dajeon, Korea) and stored at $-20^{\circ} \mathrm{C}$ until use. Each DNA sequence was brought to a stock concentration $100 \mu \mathrm{M}$ in TE buffer (10 mM Tris-HCl, $1 \mathrm{mM}$ EDTA, pH 8.0), and RNA sequences were hydrated in 
Table 1. Sequences in experiments. ${ }^{\mathrm{a}}$

\begin{tabular}{llll}
\hline & \multicolumn{1}{c}{ Case A } & & Case B \\
Name & Sequence & Name & Sequence \\
\hline$A_{1} \vee B_{1}$ & tagcgtgggtgagtacggtggttggtgtgt & $A_{1} \vee B_{1}\left(X_{1}\right)$ & caaagugcuuacagugcagguagu $^{\mathrm{b}}$ \\
& & $A_{2} \vee B_{2}\left(X_{2}\right)$ & uauugcacuugucceggccug $^{\mathrm{c}}$ \\
& & $A_{3} \vee B_{3}\left(X_{3}\right)$ & ugugcaaaucuaugcaaaacuga $^{\mathrm{d}}$ \\
\hline$A_{0} \vee B_{0}$ & atggcaacctcttcttcatcctt & $A_{0} \vee B_{0}(\mathrm{LE} 3-1)$ & atggcaacggagagagag \\
$C$ & agtcgttt-thiol & $C(\mathrm{LE} 3-2)$ & agtcgttt-thiol \\
$\neg A_{0}$ & gttgccat-thiol & $\neg A_{0}(\mathrm{LE} 3-3)$ & gttgccat-thiol \\
$\neg B_{0} \vee \neg A_{1}$ & tactcacccacgctaaaggatgaagaagag & $\neg B_{0} \vee \neg A_{1}$ (LE3-4) & tgtaagctgtttgctctctcc \\
$\neg B_{1} \vee \neg C$ & aaacgactacacaccaaccaccg & $\neg B_{1} \vee \neg A_{2}$ (LE3-5) & gacttgtgcaataactacctgcac \\
& & $\neg B_{2} \vee \neg A_{3}$ (LE3-6) & atagatttggtcacaggcttg \\
& & $\neg B_{3} \vee \neg C$ (LE3-7) & aaacgacttcagttttgc \\
\hline
\end{tabular}

\footnotetext{
a All sequences are shown in the 5' to 3' direction.

b $h s a-m i R-17-5 p$ (mirBASE accession no. MIMAT0000070).

c hsa-miR-92-1 (mirBASE accession no. MIMAT0000092).

d $h s a-m i R-19 a$ (mirBASE accession no. MIMAT0000073).
}

diethylpyrocarbonate (DEPC)-treated RNase-free water.

To cleave disulfide bonds among thiol-modified oligonucleotides, the modified sequences were treated with $100 \mathrm{mM}$ dithiothreitol (DTT) (Sigma, USA) for $10 \mathrm{~min}$. DTT was removed by extracting three times with four volumes of ethyl acetate (Sigma, USA). The mixture was vortexed for $5 \mathrm{~min}$ and centrifuged for $5 \mathrm{~min}$ at 12,000 rpm. After discarding the supernatant, the solutions were dried for $1 \mathrm{~h}$ at $37^{\circ} \mathrm{C}$ to remove the ethyl acetate completely. Water was added to a final concentration of $100 \mu \mathrm{M}$. We then immediately added thiol-tagged oligonucleotides to Au-NPs to avoid disulfide dimerizations of free-sulfhydryl groups. The final concentration of thiol-modified sequences was $1 \mu \mathrm{M}$ in colloidal Au-NP solution. The colloidal gold nanoparticles (13 $\pm 2 \mathrm{~nm} ; 1.4 \times 10^{12}$ particles $/ m \ell$ ) were purchased from BBInternational (London, UK). The mixtures were incubated for $24 \mathrm{~h}$ at room temperature with gentle agitation. They were then incubated for $40 \mathrm{~h}$ in aging buffer $(0.1 \mathrm{M} \mathrm{NaCl}, 0.1 \mathrm{M}$ sodium phosphate). The remaining sequences were removed by centrifugation (13,200 rpm, $30 \mathrm{~min}, 2$ times). The DNA-Au-NP complexes were dissolved in aging buffer to the final concentrations (about 400 strands per particle) and were stored at $4^{\circ} \mathrm{C}$ until use. The solution can be kept for several months without observing any instability.

\subsection{Hybridization and visualization}

The other sequences were added to $100 \mu \ell$ of DNA-Au-NP complex, and $\mathrm{NaCl}$ was added in $200 \mu \ell$ PCR tubes. The final concentration of each sequence was $1 \mu \mathrm{M}$, and the final $\mathrm{NaCl}$ concentration was $0.2 \mathrm{M}$. The reaction mixture was incubated at $80^{\circ} \mathrm{C}$ for $10 \mathrm{~min}$, and the temperature was steadily lowered to $25^{\circ} \mathrm{C}$ at $1^{\circ} \mathrm{C} / 30 \mathrm{sec}$ using a thermal cycler (Bio-Rad, USA). The mixture was then incubated at room temperature for $4 \mathrm{~h}$. 
All reactions were performed in triplicate with independent sample preparation, and the reliability of solutions was tested by repeating heating and cooling cycles. The result from DNA computation was characterized by ultraviolet-visible (UV/Vis) spectrometry (Nanodrop, USA) and ultra high resolution scanning electron microscopy (UHR-SEM).

\section{Results and discussion}

\subsection{DNA computing process for resolution theorem proving}

We solved a classical logic problem of resolution theorem proving by DNA computing and visualized the results using the Au-NP aggregation color change. The goal of the problem is to determine the consistency of a given set of logic formulae using resolution deduction [18]. In Boolean logic, a clause is defined as a disjunction (logical OR, V) of Boolean variables. The resolution operates with two clauses sharing one Boolean variable and its negation (logical NOT, $\neg$ ), such as $A \vee B$ and $\neg A \vee C$. From these two clauses, we can deduce a new clause that is a union of the two clauses, except for the shared variable. In the previous example, $B \vee C$ is deduced from $A \vee B$ and $\neg A \vee C$. If the set of clauses is inconsistent, repeating the resolution operation will eventually produce a special clause (an empty clause, Nil). If this does not happen, we can conclude that the original set is logically consistent. Depending on the class of the problem, resolution theorem proving may require exponential run time [19]. However, by representing a negation of a Boolean variable as the complementary sequence of the variable, the hybridization between two strands, representing the clauses, can be used for resolution theorem proving in a parallel way [20].

Here, we introduce a simple resolution theorem proving problem. Consider a set of formulae $\Delta=\left\{\neg A_{0}, A_{0} \vee B_{0}, A_{1} \vee B_{1}, A_{i} \vee B_{i}, A_{n} \vee B_{n}, \neg B_{0} \vee \neg A_{1}, \neg B_{1} \vee \neg A_{2}, \neg B_{i-1} \vee\right.$ $\left.\neg A_{i}, \neg B_{n-1} \vee \neg A_{n}, \neg B_{n} \vee \neg C, C\right\}(i=1,2, \ldots, n)$. Figure 1(a) shows an example when $n=1$. As shown in figure $1(\mathrm{c}), \Delta$ is inconsistent, and an empty clause (Nil) can be reached by resolution. The DNA computing process to solve this problem can be realized using the following principles: each different Boolean variable is encoded as a DNA sequence, its negation as a complementary sequence, and clauses as the concatenation of sequences representing the variables and negations (figure 1(a)). The hybridization of two sequences represents resolution of the two clauses. If $\Delta$ is inconsistent, the DNA hybridization will build a complete double-stranded structure (figure 1(b)). Therefore, the formation of the double-stranded structure indicates the inconsistency of $\Delta$.

\subsection{Visualizing DNA computing process using Au-NP aggregation}

The results can be easily visualized using $\mathrm{Au}-\mathrm{NP}$ aggregation. Here, the sequences for $\neg A_{0}$ and $C$ are thiol-modified and attached to Au-NPs. The double-stranded structure, if formed, would trigger the aggregation of attached Au-NPs and show a visible color change from dark red to purple [14]. If any of the clauses in $\Delta$ are missing, the empty 
(a) $X_{1} \Rightarrow A_{1} \vee B_{1}\left(A_{1} B_{1} B_{1}\right)$ $\triangle=\left\{\neg A_{0}, C, A_{0} \vee B_{0}, A_{1} \vee B_{1}\right.$, $\left.\neg B_{0} V \neg A_{1}, \neg B_{1} V \neg C\right\}$ (b)

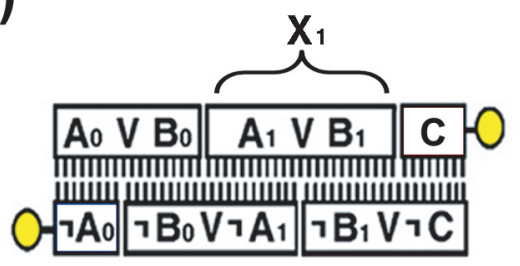

(c)

Resolution Process

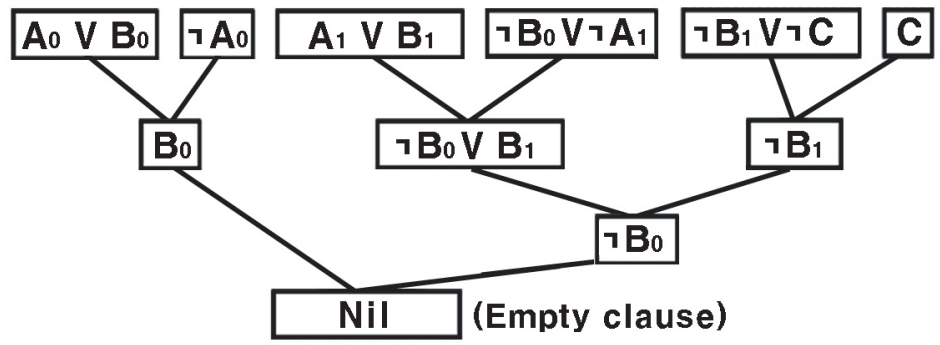

(d) DNA Computing
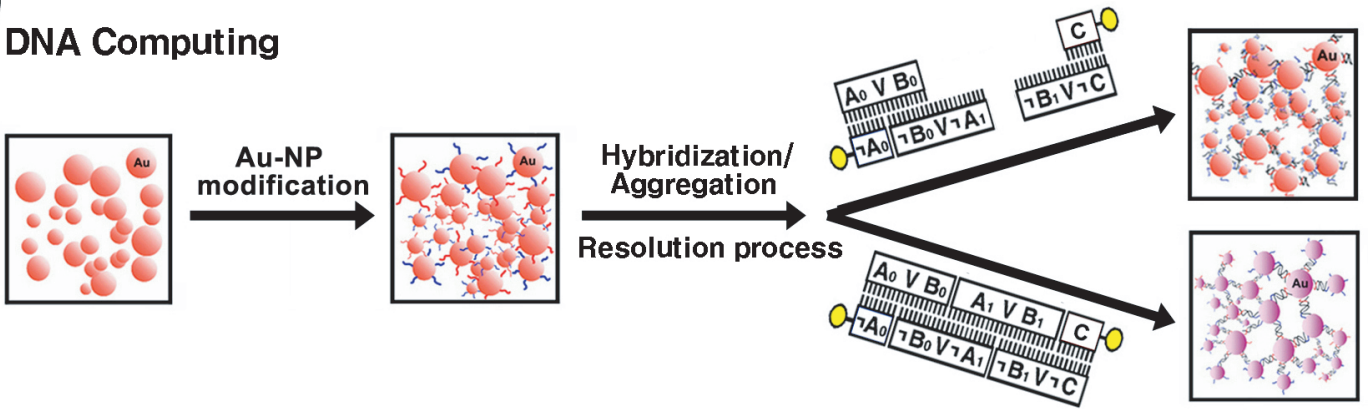

Figure 1. Schema of the proposed DNA computing process for resolution theorem proving. (a) The clauses in the example case and the encoding of clauses. A different sequence is assigned to each Boolean variable, and a negation of the variable is represented by its complementary sequence. A clause corresponds to the concatenation of the variables. (b) The final hybridized structure based on the formulae in (a). (c) Resolution theorem proving process for the set of clauses, $\Delta$, in (a). The formula connected by lines is derived from the two above by the resolution operation. For example, $B_{0}$ is derived from $A_{0} \vee B_{0}$ and $\neg A_{0}$ by the resolution. An empty clause (Nil) is derived by resolution among input clauses. (d) Visualization of the proposed DNA computing process to solve the resolution theorem proving problem using Au-NPs. DNA sequences are hybridized by complementarity-the DNA computing process-and the result indicating the empty clause induces a large three-dimensional aggregation of $\mathrm{Au}-\mathrm{NPs}$ visualized by colorimetric changes. The circle indicates the Au-NP attached to thiol-modified DNA. By replacing $A_{i} \vee B_{i}$ as the target molecules, the same method can be applied to detect other biomolecules. 
(a)

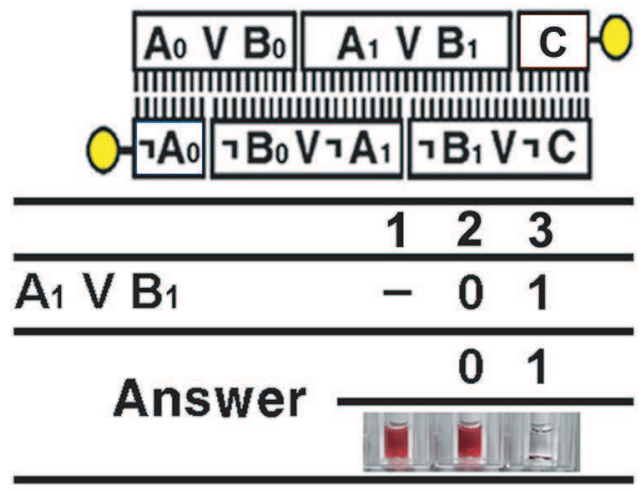

(b)

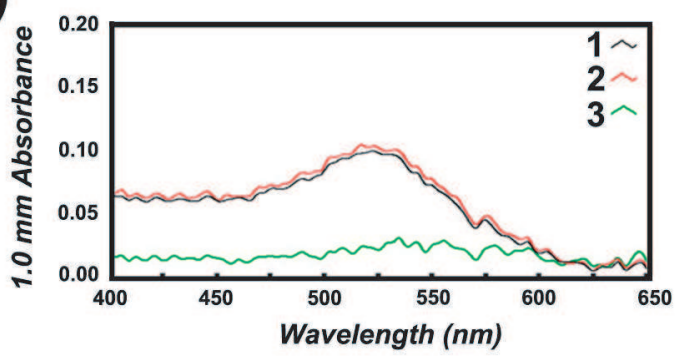

(c)

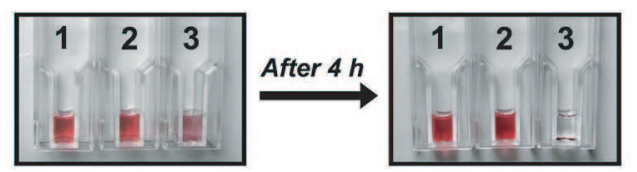

(d)
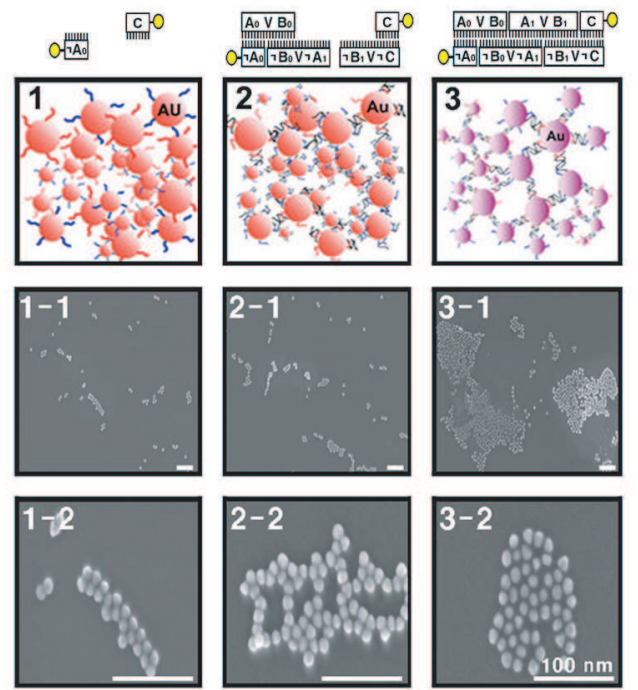

Figure 2. Visualization of DNA computing results using Au-NP aggregation. (a) The experimental results from resolution theorem proving for various cases. The top image indicates the expected structure when the given set of formulae is inconsistent. The corresponding sequences are given in table 1 (case A). Column 1 shows the control case where all sequences except for those attached to Au-NPs do not exist. The second row shows whether the clause shown on the left exists (1) or not (0). The last row shows the visualization result: no aggregation (0) and positive aggregation (1). (b) $\mathrm{UV} / \mathrm{V}$ is spectrometry results. The numbers denote the three cases in (a). Only case 3 shows the expected spectrum change. (c) The color responses of three cases in (a) over time; the tubes immediately after hybridization (left) and the same tubes after $4 \mathrm{~h}$ at room temperature (right). The numbers correspond to the three cases in (a). (d) The UHR-SEM analysis. The three cases in (a) are illustrated in the first row. The second and third rows show the UHR-SEM images under magnifications of $100 \mathrm{~K}$ and $500 \mathrm{~K}$, respectively. Bars indicate $100 \mathrm{~nm}$. The massive aggregation can be observed only in case 3 (image $3-1$ ). The aggregation shows a uniform distance between nanoparticles possibly caused by the double-stranded DNA structures (image 3-2).

clause cannot be drawn; thus the aggregation of Au-NPs and the colorimetric change will not occur. This process is summarized in figure 1(d).

\subsection{Experimental results}

We validated our method on a simple resolution theorem proving problem as shown in figure 1(a). It can be shown (figure 1(c)) that resolving the clauses eventually draws the empty clause (Nil), implying that the given set of clauses is logically inconsistent. 
The expected hybridization structure can be seen in figure 2(a). The corresponding sequences are given in table 1 (case A). However, if any of the clauses, for example $A_{1} \vee B_{1}$, is missing from $\Delta, \Delta$ is not inconsistent, and it is impossible to draw the empty clause. Therefore, depending on the consistency of $\Delta$, the color change of AuNP aggregation may or may not be observed. The experimental results are shown in figure 2(a). Column 1 indicates the control case when only Au-NPs and the attached sequences exist. The middle row indicates whether the clause $A_{1} \vee B_{1}$ exists (1) or not (0), and the bottom row (answer row) shows whether the empty clause can be drawn (1) or not (0). The tubes below the answer row show the aggregation results. We can observe that Au-NPs aggregate only when the empty clause can be drawn (column 3, the rightmost tube in figure 2(a)). Figure 2(b) shows the color changes of Au-NPs by $\mathrm{UV} / \mathrm{V}$ is spectrometry. Here, the peaks of cases 1 and 2 appear in the range of 515-525 $\mathrm{nm}$, the characteristic plasmon peak of the dark red colloidal Au-NPs ( $13 \mathrm{~nm})$, while case 3 shows a large spectrum shift. Figure 2(c) shows the color change over time. The left image was taken shortly after hybridization, and tube 3 shows a different color from the others. After $4 \mathrm{~h}$ at room temperature (right image), the aggregation proceeds, and it appears as a clear solution because of the precipitation of large DNA-Au-NP complexes.

A more detailed analysis using UHR-SEM (figure 2(d)) confirms the results in figure 2(c). The results from DNA computing for resolution theorem proving can be categorized into three classes. The first class consists of only Au-NPs conjugated with thiol-tagged DNAs where no resolution can be applied, and consequently, the aggregation is impossible. The second is the case when the set of clauses is consistent because some clauses needed to draw the empty clause are missing. This case is also unable to aggregate and shows no colorimetric changes because the missing DNAs cause a defect in hybridization. The third class is when there is no missing clause. Here, the DNAs can be fully linked and form large Au-NP aggregates, indicating that the resolution process produced an empty clause. For figure 2(a), the first class corresponds to the control case (column 1), the second to column 2 and the third to column 3. For the first and second classes, such large networks of Au-NPs are not observed (1-1 and 2-1 in figure 2(d)), and the interparticle distance is almost zero (1-2 and 2-2 in figure $2(\mathrm{~d}))$. For the third class, the image shows an extensively cross-linked network of Au-NPs (3-1 in figure 2(d)). With an enlarged view (3-2 in figure 2(d)), the network consists of the close-packed Au-NPs with uniform distances produced by hybridization of DNAs attached to nanoparticles $[14,21]$. The size of this network grows significantly after $4 \mathrm{~h}$ and causes precipitation as shown in figure $2(\mathrm{c})$.

\subsection{Application to biomolecular detection}

As mentioned in section 3.2, the aggregation of Au-NPs will not happen if the clauses in $\Delta$ necessary to deduce the empty clause are missing ( $\Delta$ is not inconsistent). Using this property, we applied the same procedure to detect multiple target molecules. Depending 
(a)

Application of resolution refutation for biomolecular detection

\section{Biological target data \\ (e.g. microRNAs)}

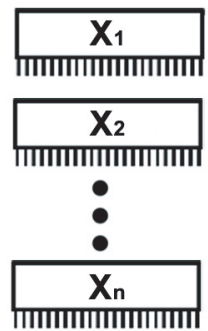

(b)

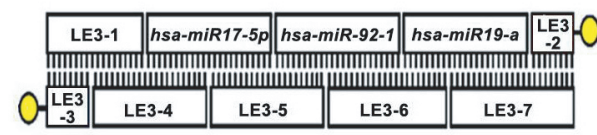

Construct DNA logic elements (LEs)

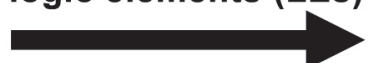

Target data

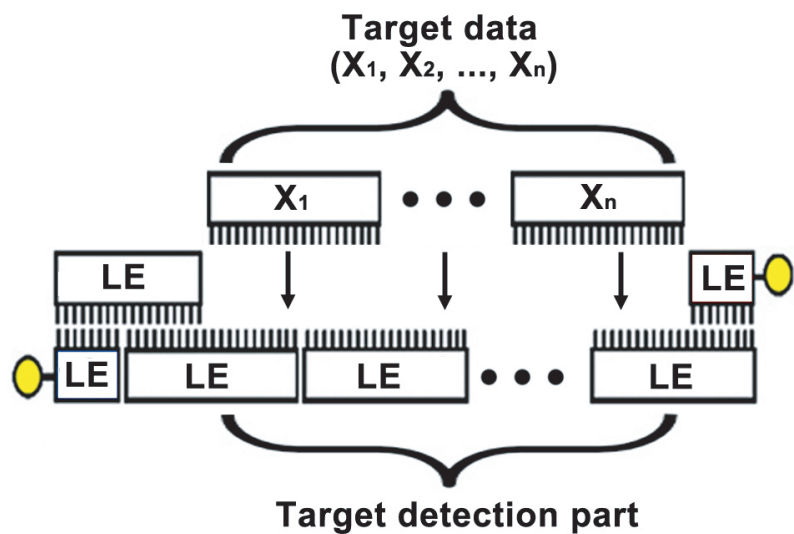

Figure 3. Application of resolution theorem proving to logic-based biomolecular detection. (a) With each target molecule $\left(X_{i}\right)$ as a clause, we construct a simple problem where the empty clause (double-stranded structure) is produced only when all targets exist. (b) The experimental results for three target miRNAs. The left figure indicates the expected structure when all targets exist. The corresponding sequences are given in table 1 (case B). Column $\mathrm{C}$ shows the control case where all sequences, except for those attached to $\mathrm{Au}-\mathrm{NPs}$, do not exist. The second row shows that the target molecule shown on the left exists (1) or not (0). The last row shows the visualization result: no aggregation (0) and positive aggregation (1). The existence of multiple targets can be visualized in one tube.

on the number of targets, we constructed $\Delta$ so that it was consistent if any of the targets was missing. Suppose we have $n$ targets, $X_{1}, X_{2}, \ldots, X_{n}$. The left and right half of each $X_{i}$ can be used as Boolean variables $A_{i}$ and $B_{i}$, respectively, and consequently, $X_{i}$ can be interpreted as encoding $A_{i} \vee B_{i}$. The other clauses in $\Delta$ are coded into DNA sequences according to $X_{i}$ 's. In this way, the absence of any target would make $\Delta$ consistent and prevent the aggregation of Au-NPs. Therefore, one can judge the coexistence of all targets from the aggregation of Au-NPs (figure 3(a)). Among the various types of nucleic acids, we chose miRNA as a target for its small size ( $\sim 22 \mathrm{nt})$ and the importance of its role as a genetic regulator $[22,23]$. They are also expressed polycistronically and act cooperatively to regulate mRNA translation [24, 25]. Considering these points, detecting multiple miRNAs based on logic can improve analysis efficiency. Recently, 
miRNA recognition through DNA-based digital logic circuit operation was reported [12], which implies the possibility and usefulness of a logic-based smart analyzing system. In addition, our approach is not limited to miRNAs and can overcome the conventional analysis techniques that have many difficulties in handling small nucleic acids with cooperative expression behavior.

We used three miRNAs in the miR-17-92 cluster [24] as targets. In the cluster, there are seven miRNAs, and their expression level is increased in human B-cell lymphomas [24]. In cancer sample, all miRNAs would exist, and consequently, the Au-NP aggregation would be observed. We used only three miRNAs (miR-17-5p $\left(X_{1}\right)$, miR-92-1 $\left(X_{2}\right)$ and miR-19a $\left(X_{3}\right)$ ) of the seven to minimize the cross-hybridization between miRNA sequences. In this case, eight combinations were possible from three miRNAs. Figure 3(b) shows that the Au-NP aggregation was observed only when all three miRNAs existed (column 8). The sequences of the miRNAs and related DNAs are shown in table 1 (case B). Although we cannot identify the missing miRNA by the result, we can still benefit from the short detection time (within 5 min by UV/Vis spectrometry) and the efficiency of handling multiple targets simultaneously.

\subsection{Discussion}

We have shown that the proposed method is applicable to a problem of 3 targets and 7 logical elements (a total of 10 fragments to induce aggregation). In [26] we have additionally verified that our method can be extended to the size of 5 targets and 9 logical elements (a total of 14 fragments to induce aggregation). Several ways can be taken to further increase the problem size. One way is to minimize the cross-hybridization among the involved sequences so that more targets can be handled simultaneously. Although the cross-hybridization can happen more often for larger applications, various techniques and tools studied in fields of bioinformatics [27, 28] and DNA computing [17, 29, 30] can be used to keep the undesired hybridization to a reasonable level. Another way is to partition the targets into several groups so that the cross-hybridization inside each group is minimized and to apply separate aggregation process for each group of targets. In this way, assuming 5 or 6 targets in each group, $5 n$ or $6 n$ ( $n$ denotes the number of groups) can be dealt by the proposed method. The number of targets handled in each group can be increased further either by designing sequences carefully or by utilizing various modified nanoparticles. Yet another approach is to develop an automated system using, for example, lab-on-a-chip technology to partition the targets into non-hybridizing groups by design. If the problem structure allows this partitioning, the size of the problem to be solved can further be increased.

\section{Conclusion}

We showed that Au-NP aggregation induced by DNA hybridization and the resulting colorimetric change can visualize the results of DNA computation. Compared with the 
conventional method in DNA computing, our method reduces the monitoring time for the computation results and improves the efficiency by using a colorimetric reporter. With careful sequence design, the suggested method can be applied to a larger problem than reported here. We also demonstrated that the same technique could be used to detect multiple miRNAs simultaneously. Nanoparticles have recently been used for multiplexed assays with various fluorescent dye conjugation or size-based nanoparticle coupling [31, 32, 33, 34]. However, the multiplexibility of these techniques depends on how many types of modified nanoparticles are available rather than the number of target biomolecules to be assayed. On the other hand, advantages in the multiplexibility of input molecules over the number of nanoparticle variations are observed by applying concepts of logic and computation to analysis strategies. In this respect, our study broadens the application area of Au-NPs to a visualization tool for DNA computing and multiplexed biological assays combined with DNA computing.

\section{Acknowledgments}

This work was supported in part by Ministry of Commerce, Industry and Energy through the Molecular Evolutionary Computing (MEC) Project and by Ministry of Education and Human Resources Development (MOEHRD) under the BK21-IT Program. The ICT at Seoul National University provided research facilities for this study. KyungAe Yang was supported by Korea Research Foundation Grant funded by the Korean Government (MOEHRD, Basic Research Promotion) (KRF-2006-351-C00045).

\section{References}

[1] Adleman L 1994 Science 266 1021-4

[2] Braich R S, Chelyapov N, Johnson C, Rothemund P W K and Adleman L 2002 Science 296 499-502

[3] Lipton R J 1995 Science 268 542-5

[4] Faulhammer D, Cukras A R, Lipton R J and Landweber L F 2000 Proceedings of the National Academy of Sciences $\mathbf{9 7}$ 1385-9

[5] Macdonald J, Li Y, Sutovic M, Lederman H, Pendri K, Lu W, Andrews B L, Stefanovic D and Stojanovic M N 2006 Nano Letters 6 2598-603

[6] Stojanovic M N and Stefanovic D 2003 Nature Biotechnology 21 1069-74

[7] Lee J Y, Shin S Y, Park T H and Zhang B T 2004 BioSystems 78 39-47

[8] Sakakibara Y 2003 Natural Computing 2 153-71

[9] Zhang B T and Kim J K 2006 Proc. 12th Int. Meeting on DNA Computing (Seoul, Korea, 5-9 June 2006) (Lecture Notes in Computer Science vol 4287) ed C Mao and T Yokomori (Berlin: Springer-Verlag) pp 298-307

[10] Benenson Y, Gil B, Ben-Dor U, Adar R and Shapiro E 2004 Nature 429 423-9

[11] Rinaudo K, Bleris L, Maddamsetti R, Subramanian S, Weiss R and Benenson Y 2007 Nature Biotechnology 25 795-801

[12] Seelig G, Soloveichik D, Zhang D Y and Winfree E 2006 Science 314 1585-8

[13] Elghanian R, Storhoff J J, Mucic R C, Letsinger R L and Mirkin C A 1997 Science 277 1078-81

[14] Mirkin C A, Letsinger R L, Mucic R C and Storhoff J J 1996 Nature 382 607-9

[15] Katz E and Willner I 2004 Angewandte Chemie International Edition 43 6042-108 
[16] Thaxton C S, Georganopoulou D G and Mirkin C A 2005 Clinica Chimica Acta 363 120-6

[17] Shin S Y, Lee I H, Kim D and Zhang B T 2005 IEEE Transactions on Evolutionary Computation 9 143-58

[18] Nilsson N J 1998 Artificial Intelligence: A New Synthesis (USA: Morgan Kaufmann Publishers, Inc.)

[19] Galil Z 1974 On the complexity of resolution procedures for theorem proving Technical Report (UMI Order Number: TR74-223) Cornell University

[20] Lee I H, Park J Y, Jang H M, Chai Y G and Zhang B T 2003 Proc. 8th Int. Meeting on DNA based Computers (Hokkaido, Japan, 10-13 June 2002) (Lecture Notes in Computer Science vol 2568) ed M Hagiya and A Ohuchi (Berlin: Springer-Verlag) pp 156-67

[21] Jin R, Wu G, Li Z, Mirkin C A and Schatz G C 2003 Journal of the American Chemical Society 125 1643-54

[22] Bartel D P 2004 Cell 116 281-97

[23] Mattick J S 2003 BioEssays 25 930-9

[24] He L, Thomson J M, Hemann M T, Hernando-Monge E, Mu D, Goodson S, Powers S, CordonCardo C, Lowe S W, Hannon G J and Hammond S M 2005 Nature 435 828-33

[25] Hobert O 2004 Trends in Biochemical Sciences 29 462-8

[26] Yang K A, Shin S Y, Lee I H, Lee J H and Zhang B T 2007 Proc. 5th Int. Nanotech Symposium \& Exhibition in Korea (KINTEX, Korea, 29-31 August 2007) p 216

[27] Chen S H, Lo C Z, Tsai M C, Hsiung C A and Lin C Y 2008 BMC Bioinformatics 9 S8

[28] Feng S and Tillier E R 2007 Bioinformatics 23 1195-202

[29] Tanaka F, Kameda A, Yamamoto M and Ohuchi A 2005 Nucleic Acids Research 33 903-11

[30] Dirks R M, Lin M, Winfree E and Pierce N A 2004 Nucleic Acids Research 32 1392-403

[31] Bao Y P, Huber M, Wei T F, Marla S S, Storhoff J J and Muller U R 2005 Nucleic Acids Research 33 e15

[32] von Maltzahn G, Harris T J, Park J H, Min D H, Schmidt A J, Sailor M J and Bhatia S N 2007 Journal of the American Chemical Society 129 6064-5

[33] Stoeva S I, Lee J S, Thaxton C S and Mirkin C A 2006 Angewandte Chemie International Edition $453303-6$

[34] Wang L, O'Donoghue M B and Tan W 2006 Nanomedicine 1 413-26 